PROCEEDINGS OF THE

AMERICAN MATHEMATICAL SOCIETY

Volume 140, Number 9, September 2012, Pages 3091-3100

S 0002-9939(2012)11139-6

Article electronically published on January 4, 2012

\title{
THE ZEROS OF CERTAIN LOMMEL FUNCTIONS
}

\author{
STAMATIS KOUMANDOS AND MARTIN LAMPRECHT
}

(Communicated by Walter Van Assche)

ABSTRACT. Lommel's function $s_{\mu, \nu}(z)$ is a particular solution of the differential equation $z^{2} y^{\prime \prime}+z y^{\prime}+\left(z^{2}-\nu^{2}\right) y=z^{\mu+1}$. Here we present estimates and monotonicity properties of the positive zeros of $s_{\mu-1 / 2,1 / 2}(z)$ when $\mu \in(0,1)$. The positivity of a closely related integral is also considered.

\section{INTRODUCTION}

Lommel's function [10, Sec. 10.7]

$$
s_{\mu, \nu}(z)=\frac{z^{\mu+1}}{4} \sum_{n=0}^{\infty} \frac{(-1)^{n}\left(\frac{1}{2} z\right)^{2 n} \Gamma((\mu-\nu+1) / 2) \Gamma((\mu+\nu+1) / 2)}{\Gamma((\mu-\nu+2 n+3) / 2) \Gamma((\mu+\nu+2 n+3) / 2)}
$$

is a particular solution of the inhomogeneous Bessel equation

$$
z^{2} y^{\prime \prime}+z y^{\prime}+\left(z^{2}-\nu^{2}\right) y=z^{\mu+1}
$$

and occurs in several places in physics and engineering (see [3] for a list of references). In 9] Steinig examined the sign of $s_{\mu, \nu}(z)$ for positive $z$ and $\mu, \nu \in \mathbb{R}$. Among other things, he showed that for $\mu<\frac{1}{2}$ the function $s_{\mu, \nu}(z)$ has infinitely many changes of sign on $(0, \infty)$. Here we present the following refinement of this result. In this paper the zeros of analytic functions will always be counted according to their multiplicities and $\mathbb{N}$ will denote the set of positive integers.

Theorem 1.1. For $\mu \in(0,1)$ and $n \in \mathbb{N}$ set

$$
I_{2 n-1}(\mu):=\left(\left(2 n-1+\frac{\mu}{2}\right) \pi,(2 n-1+\mu) \pi\right]
$$

and

$$
I_{2 n}(\mu):=\left(2 n \pi,\left(2 n+\frac{\mu}{2}\right) \pi\right) .
$$

Then, for $\mu \in(0,1)$ and positive $z, s_{\mu-1 / 2,1 / 2}(z)=0$ implies $z \in I_{n}(\mu)$ for an $n \in \mathbb{N}$ and $s_{\mu-1 / 2,1 / 2}(z)$ has exactly one zero in every interval $I_{n}(\mu)$.

Hence, for each $n \in \mathbb{N}$ and $\mu \in(0,1)$ there is a $z_{n}:=z_{n}(\mu) \in I_{n}$ such that $s_{\mu-1 / 2,1 / 2}\left(z_{n}\right)=0$, and this $z_{n}$ satisfies $s_{\mu-1 / 2,1 / 2}^{\prime}\left(z_{n}\right) \neq 0$. The implicit function theorem therefore implies that each $z_{n}$ is an analytic function of $\mu \in(0,1)$.

Received by the editors October 8, 2010 and, in revised form, March 21, 2011.

2010 Mathematics Subject Classification. Primary 33C10, 33B10; Secondary 42A05, 30C15, $26 \mathrm{D} 15$.

Key words and phrases. Special functions, Lommel functions, positive integrals, zeros.

The research for this paper was supported by the Leventis Foundation (grant No. 3411-21041).

(C)2012 American Mathematical Society Reverts to public domain 28 years from publication 
Theorem 1.2. Set $w_{n}:=w_{n}(\mu):=z_{n}-n \pi$. For $\mu \in(0,1)$ we have $\lim _{n \rightarrow \infty} w_{n}=$ $\mu \pi / 2$ and

$$
w_{2 n}<w_{2 n+2}<\frac{\mu \pi}{2}<w_{2 n+1}<w_{2 n-1}, \quad n \in \mathbb{N} .
$$

Furthermore, for $n \in \mathbb{N}$ the functions $z_{2 n-1}$ are strictly increasing in $(0,1)$.

Proposition 1.4 below shows that $z_{2 n}$ is not monotonic in $\mu$ for any $n \in \mathbb{N}$.

Our proofs of Theorems 1.1 and 1.2 will be based on the fact that for $\mu, \nu \in \mathbb{C}$ with $\Re(\mu \pm \nu+1)>0$ and $z \in \mathbb{C}^{-}:=\mathbb{C} \backslash\{z: z \leq 0\}$, one has

$$
s_{\mu, \nu}(z)=\frac{\pi}{2}\left[Y_{\nu}(z) \int_{0}^{z} t^{\mu} J_{\nu}(t) d t-J_{\nu}(z) \int_{0}^{z} t^{\mu} Y_{\nu}(t) d t\right],
$$

where $J_{\nu}(z)$ and $Y_{n}(z)$ are the usual Bessel functions [10, Sec. 10.7]. It is wellknown that

$$
J_{1 / 2}(z)=\sqrt{\frac{2}{\pi z}} \sin z \quad \text { and } \quad Y_{1 / 2}(z)=-\sqrt{\frac{2}{\pi z}} \cos z,
$$

and therefore we obtain from (1.2) that for $\mu>0$,

$$
s_{\mu-1 / 2,1 / 2}(z)=\frac{1}{z^{1 / 2}} \int_{0}^{z} t^{\mu-1} \sin (z-t) d t .
$$

Theorem 1.1 thus takes the following equivalent form.

Theorem 1.3. For all $\mu \in(0,1)$ the function

$$
F_{\mu}(z):=\int_{0}^{z} t^{\mu-1} \sin (z-t) d t
$$

has exactly one zero in each interval $I_{n}(\mu), n \in \mathbb{N}$, and vanishes nowhere else on the positive real axis.

Obviously, $F_{\mu}(z)$ is analytic for $\Re \mu>0$ and $z \in \mathbb{C}^{-}$. Partial integration shows that

$$
F_{\mu}(z)=\frac{1}{\mu} \int_{0}^{z} t^{\mu} \cos (z-t) d t=: \frac{1}{\mu} G_{\mu}(z) .
$$

The following proposition is thus clear.

Proposition 1.4. For $n \in \mathbb{N}$ we have $z_{n} \rightarrow n \pi$ as $\mu \rightarrow 0$ and $z_{2 n-1}(1)=z_{2 n}(1)=$ $2 n \pi$.

Our interest in the Lommel functions $s_{\mu-1 / 2,1 / 2}(z)$ stems from the fact that the function $F_{\mu}(z)$ plays an important role in the theory of positive trigonometric sums.

In [11, V.2.29] it is shown that there is exactly one $\alpha^{*} \in(0,1)$ such that $F_{1-\alpha^{*}}\left(\frac{3 \pi}{2}\right)$ vanishes. In [2] it is proven that $\alpha^{*}$ is such that for all $\alpha \geq \alpha^{*}, n \in \mathbb{N}$, and $x \in(0, \pi)$,

$$
\tau_{n}(\alpha, x):=1+\sum_{k=1}^{n} \frac{\cos (k x)}{k^{\alpha}}>0,
$$

while for $\alpha<\alpha^{*}$ the cosine polynomials $\tau_{n}(\alpha, x)$ do not have a uniform lower bound on $(0, \pi)$. Nowadays, $\alpha^{*}=0.30844 \ldots$ is called the Littlewood-Salem-Izumi constant [1]. See also [4 and [6] for related results and considerations. 
In [7] it is claimed that for each $\rho \in(0,1)$ there is exactly one $\mu^{*}(\rho) \in(0,1)$ such that

$$
F_{\mu^{*}(\rho)}((\rho+1) \pi)=0 .
$$

It is then shown there that the thus defined function $\mu^{*}(\rho)$ plays an important role in a conjecture concerning the mapping properties of partial sums of certain univalent functions. In [5] it is proven that this conjecture is equivalent to the fact that for $n \in \mathbb{N}, \rho \in(0,1)$, and $\theta \in(0, \pi)$ one has

$$
\sum_{k=0}^{n} \frac{(\mu)_{k}}{k !} \sin [(2 k+\rho) \theta]>0
$$

for all $\mu \in\left(0, \mu^{*}(\rho)\right]$ but for no $\mu \in\left(\mu^{*}(\rho), 1\right)$. Here, $(\mu)_{k}:=\mu(\mu+1) \cdots(\mu+k-1)$ is the Pochhammer symbol.

It seems that in 7 the proof of the well-definition of $\mu^{*}(\rho)$ is not completely correct. In order to prove that for each $\rho \in(0,1)$ there is exactly one $\mu^{*}(\rho) \in(0,1)$ such that (1.4) holds, it is claimed in the proof of [7, Lem. 1] that for all $\rho, \mu \in(0,1)$,

$$
I(\rho, \mu):=\frac{d}{d \mu} F_{\mu}((\rho+1) \pi)=\int_{0}^{(\rho+1) \pi} t^{\mu-1} \sin ((\rho+1) \pi-t) \log (t) d t>0 .
$$

But for $\rho=0$ it is easy to see that

$$
I(0, \mu)=\int_{0}^{\pi} t^{\mu-1} \sin (t) \log (t) d t
$$

takes a negative value when $\mu=0$. Hence, because of continuity, there is an $\epsilon>0$ such that for all $\mu \in(0, \epsilon)$ there is a $\rho>0$ for which $I(\rho, \mu)<0$.

Despite this, the function $\mu^{*}(\rho)$ is well-defined, as we can show here. It follows readily from Theorem 1.2 and Proposition 1.4 that $z_{1}$ is a strictly increasing analytic function from $(0,1)$ onto $(\pi, 2 \pi)$. Hence, $\mu^{*}(\rho)$ is well-defined, and we have $\mu^{*}(\rho)=$ $z_{1}^{(-1)}((\rho+1) \pi)$. The next proposition is thus clear.

Proposition 1.5. For each $\rho \in(0,1]$ there is exactly one $\mu^{*}(\rho) \in(0,1]$ such that $F_{\mu^{*}(\rho)}((\rho+1) \pi)=0 . \mu^{*}(\rho)$ is strictly increasing and analytic in $(0,1)$.

In order to prove our results we will first show a weak form of Theorem 1.3 in the next section. In Section 3 we will then present the proofs of Theorems 1.2 and 1.3 . For the proof of Theorem 1.2 we will need some information concerning the set of $(\rho, \mu) \in(0,1)^{2}$ for which $I(\rho, \mu)$ is positive. The relevant results will be presented in Section 4.

\section{A WEAK FORM OF ThEOREM 1.3}

In this section we will prove the following lemma.

Lemma 2.1. For $\mu \in(0,1)$ the function $F_{\mu}(z)$ has exactly one zero in every interval $(n \pi,(n+1) \pi), n \in \mathbb{N}$, and vanishes nowhere else on the positive axis.

First, note that, since $t^{\mu-1}$ is decreasing on $(0, \infty)$ when $\mu \in[0,1)$, obviously

$$
\int_{0}^{a} t^{\mu-1} \sin (t) d t>0 \quad \text { when } \quad a>0 \text { and } \mu \in[0,1)
$$

The next lemma is the key result for the proof of Lemma 2.1. 
Lemma 2.2. Suppose $\mu \in(0,1)$ and $z^{*}>0$ are such that $F_{\mu}\left(z^{*}\right)=0$. Then

$$
F_{\mu}^{\prime}\left(z^{*}\right)=\int_{0}^{z^{*}} t^{\mu-1} \cos \left(z^{*}-t\right) d t \neq 0 .
$$

Proof. If both $F_{\mu}\left(z^{*}\right)$ and $F_{\mu}^{\prime}\left(z^{*}\right)$ would vanish, then so would

$$
F_{\mu}\left(z^{*}\right)+i F_{\mu}^{\prime}\left(z^{*}\right)=e^{i z^{*}} \int_{0}^{z^{*}} t^{\mu-1} e^{-i t} d t .
$$

In particular,

$$
\int_{0}^{z^{*}} t^{\mu-1} \sin (t) d t=0
$$

would hold. Since this contradicts (2.1), the proof is complete.

It follows from (2.1) that $F_{\mu}(n \pi) \neq 0$ and

$$
\operatorname{sgn} F_{\mu}(n \pi)=(-1)^{n+1} \text { for } \quad \mu \in(0,1), n \in \mathbb{N} .
$$

Furthermore, it is easy to see that $F_{\mu}(z)$ is positive for $z \in(0, \pi)$ and $\mu \in(0,1)$.

Now, in order to verify the remaining statements of Lemma 2.1, let $n \in \mathbb{N}$ and $\mu^{*} \in(0,1)$ and suppose that $F_{\mu^{*}}$ has exactly $m \in\{1,2,3, \ldots\}$ zeros in the interval $I:=(n \pi,(n+1) \pi)$. Then, because of Lemma 2.2, $F_{\mu^{*}}$ has $m$ simple zeros $x_{1}, \ldots, x_{m}$ in $I$ and therefore there are $m$ open subintervals $J_{1}, \ldots, J_{m}$ of $(0,1)$, all containing $\mu^{*}$, and $m$ differentiable functions $x_{k}(\mu), k \in\{1, \ldots, m\}$, that satisfy $x_{k}\left(\mu^{*}\right)=x_{k}$ and $F_{\mu}\left(x_{k}(\mu)\right)=0$ for $\mu \in J_{k}$. Since $F_{\mu}(n \pi) \neq 0$ for all $\mu \in(0,1)$ and $n \in \mathbb{N}, x_{k}(\mu)$ lies in $I$ for all $\mu \in J_{k}$ and $k \in\{1, \ldots, m\}$.

If $k \in\{1, \ldots, m\}$ and $\sigma \in(0,1)$ is a boundary point of $J_{k}$, then $\lim _{\mu \rightarrow \sigma} x_{k}(\mu)$ exists. Otherwise, because $x_{k}(\mu)$ is continuous and satisfies $x_{k}(\mu) \in I$ for $\mu \in J_{k}$, there is an open non-empty subinterval $I^{*}$ of $I$ such that for every $z \in I^{*}$ the set $x_{k}^{(-1)}(z)$ contains an infinite number of points that accumulate at $\sigma$. Consequently, $F_{\sigma}(z)=0$ for all $z \in I^{*}$, a contradiction.

We can therefore assume that $J_{k}=(0,1)$ for all $k \in\{1, \ldots, m\}$. Otherwise, because of what we have just shown, one interval $J_{k}$ would have a boundary point $\sigma \in(0,1)$ for which

$$
F_{\sigma}\left(\lim _{\mu \rightarrow \sigma} x_{k}(\mu)\right)=0=F_{\sigma}^{\prime}\left(\lim _{\mu \rightarrow \sigma} x_{k}(\mu)\right) .
$$

This would contradict Lemma 2.2

We have thus proven that if $F_{\mu}$ has exactly $m$ zeros in $(0,1)$ for one $\mu \in(0,1)$, then $F_{\mu}$ has at least $m$ zeros in $(0,1)$ for all $\mu \in(0,1)$. This readily implies that if $F_{\mu}$ has exactly $m$ zeros in $(0,1)$ for one $\mu \in(0,1)$, then $F_{\mu}$ has exactly $m$ zeros in $(0,1)$ for all $\mu \in(0,1)$.

In order to complete the proof of Lemma 2.1 we will now determine a $\mu \in(0,1)$ for which $F_{\mu}$ has exactly one zero in $I$.

To that end, observe that, because of (1.3), $F_{\mu}$ vanishes if, and only if, $G_{\mu}$ vanishes. For positive $z$ the function $G_{0}(z)=\sin (z)$ vanishes exactly at the points $n \pi, n \in \mathbb{N}$, and satisfies $G_{0}^{\prime}(n \pi)=(-1)^{n}$. Therefore for every $n \in \mathbb{N}$ there is a differentiable function $z_{n}(\mu)$, defined in an open real neighborhood $U_{n}$ of the origin, such that $z_{n}(0)=n \pi, G_{\mu}\left(z_{n}(\mu)\right)=0$ for all $\mu \in U_{n}$, and

$$
z_{n}^{\prime}(0)=-\frac{\left.\frac{d}{d \mu} G_{\mu}\left(z_{n}(0)\right)\right|_{\mu=0}}{G_{0}^{\prime}\left(z_{n}(0)\right)}=(-1)^{n+1} \int_{0}^{n \pi} \cos (n \pi-t) \log (t) d t .
$$


Partial integration gives

$$
\int_{0}^{n \pi} \cos (n \pi-t) \log (t) d t=(-1)^{n+1} \int_{0}^{n \pi} \frac{\sin (t)}{t} d t
$$

The integral on the right-hand side of this equation is positive because of (2.1), and thus it follows from (2.3) and Hurwitz's theorem that for every $n \in \mathbb{N}$ there is a $\mu_{n}>0$ such that for $0<\mu<\mu_{n}$ the functions $G_{\mu}$ and $F_{\mu}$ have exactly one zero in $(n \pi,(n+1) \pi)$. The proof of Lemma 2.1 is complete.

\section{Proof of Theorems 1.2 and 1.3}

The following result is an easy consequence of Lemma 2.1 and (2.2).

Proposition 3.1. For a $z \in(n \pi,(n+1) \pi)$ the relations

$$
F_{\mu}(z)>0 \text { and } F_{\mu}(z)<0
$$

hold if, and only if, $z<z_{n}$ and $z>z_{n}$, respectively, in the case where $n$ is odd, and if, and only if, $z>z_{n}$ and $z<z_{n}$, respectively, in the case where $n$ is even.

Now, let $n \in \mathbb{N}, n \geq 3$. Then

$$
\begin{aligned}
0 & =\int_{0}^{z_{n}} t^{\mu-1} \sin \left(z_{n}-t\right) d t \\
& =\int_{0}^{z_{n}-2 \pi} t^{\mu-1} \sin \left(z_{n}-t\right) d t+\int_{z_{n}-2 \pi}^{z_{n}} t^{\mu-1} \sin \left(z_{n}-t\right) d t
\end{aligned}
$$

Since $t^{\mu-1}$ is decreasing on $(0, \infty)$, it is clear that the integral on the right-hand side of the sum is negative, and therefore

$$
\int_{0}^{z_{n}-2 \pi} t^{\mu-1} \sin \left(z_{n}-2 \pi-t\right) d t>0
$$

Hence, it follows from Proposition 3.1 that

$$
z_{n}-2 \pi<z_{n-2} \quad \text { or } \quad z_{n}-2 \pi>z_{n-2},
$$

depending on whether $n$ is odd or even.

We have thus shown that, for fixed $\mu \in(0,1)$, the sequence $w_{2 n-1}$ is strictly decreasing, while $w_{2 n}$ is strictly increasing. Consequently, since $w_{n} \in(0, \pi)$ for all $n \in \mathbb{N}, w_{e}=\lim _{n \rightarrow \infty} w_{2 n}$ and $w_{o}=\lim _{n \rightarrow \infty} w_{2 n-1}$ exist.

In order to prove that $w_{e}=w_{o}=\mu \pi / 2$, recall that the generalized sine and cosine integrals are defined by

$$
\operatorname{Si}(z, \mu):=\int_{0}^{z} t^{\mu-1} \sin (t) d t \quad \text { and } \quad \operatorname{Ci}(z, \mu):=\int_{0}^{z} t^{\mu-1} \cos (t) d t,
$$

respectively, and satisfy

$$
\lim _{z \rightarrow \infty} \operatorname{Si}(z, \mu)=\sin \left(\frac{\mu \pi}{2}\right) \Gamma(\mu) \quad \text { and } \quad \lim _{z \rightarrow \infty} \operatorname{Ci}(z, \mu)=\cos \left(\frac{\mu \pi}{2}\right) \Gamma(\mu)
$$

for $z$ tending to $\infty$ on the real axis and $\mu \in(0,1)$ []. For all $n \in \mathbb{N}$ we have

$$
0=(-1)^{n} F_{\mu}\left(z_{n}\right)=\sin \left(w_{n}\right) \operatorname{Ci}\left(z_{n}, \mu\right)-\cos \left(w_{n}\right) \operatorname{Si}\left(z_{n}, \mu\right) .
$$

Letting $n \rightarrow \infty$, we obtain from (3.1) that both for $w=w_{e}$ and $w=w_{o}$,

$$
0=\Gamma(\mu) \sin \left(w-\frac{\mu \pi}{2}\right)
$$

and thus that $w_{e}=w_{o}=\mu \pi / 2$. 
Next, observe that, according to the implicit function theorem,

$$
z_{n}^{\prime}=-\frac{\left.\frac{d}{d \sigma} F_{\sigma}\left(z_{n}\right)\right|_{\sigma=\mu}}{F_{\mu}^{\prime}\left(z_{n}\right)} .
$$

Since $F_{\mu}^{\prime}\left(z_{n}\right)$ does not change sign in $(0,1)$, it follows from Proposition 3.1 that in order to prove that $z_{2 n-1}$ is increasing in $(0,1)$, it will suffice to show

$$
\left.\frac{d}{d \sigma} F_{\sigma}\left(z_{2 n-1}\right)\right|_{\sigma=\mu}=\int_{0}^{z_{2 n-1}} t^{\mu-1} \log (t) \sin \left(t-w_{2 n-1}\right) d t>0
$$

for $\mu \in(0,1)$.

To that end, note first that

$$
\begin{aligned}
\int_{0}^{z_{1}} t^{\mu-1} \log (t) \sin \left(t-w_{1}\right) d t= & \int_{0}^{w_{1}} t^{\mu-1} \log (t) \sin \left(t-w_{1}\right) d t \\
& +\int_{w_{1}}^{z_{1}} t^{\mu-1} \log (t) \sin \left(t-w_{1}\right) d t .
\end{aligned}
$$

Since $t^{\mu-1} \sin \left(t-w_{1}\right)<0$ and $t^{\mu-1} \sin \left(t-w_{1}\right)>0$ for $t \in\left(0, w_{1}\right)$ and $t \in\left(w_{1}, z_{1}\right)$, respectively, we therefore obtain

$$
\int_{0}^{z_{1}} t^{\mu-1} \log (t) \sin \left(t-w_{1}\right) d t>\log \left(w_{1}\right) F_{\mu}\left(z_{1}\right)=0 .
$$

We have thus shown that $z_{1}$ is a strictly increasing and analytic function in $(0,1)$. As explained in the paragraph preceding Proposition 1.5. this implies that the function $\mu^{*}(\rho)$ is well-defined. We can therefore make use of the inequality

$$
\rho \leq \mu^{*}(\rho), \quad \rho \in(0,1),
$$

which was established in 7 .

Now, before we show (3.2) also for $n=2,3, \ldots$, we will first complete the proof of Theorem 1.3

Proof of Theorem 1.3. Because of Proposition 3.1 and what we have shown so far, only the inequality $F_{\mu}((2 n-1+\mu) \pi) \leq 0, n \in \mathbb{N}$, remains to be verified. In fact, since

$$
\begin{aligned}
F_{\mu}((2 n-1+\mu) \pi) & =F_{\mu}((1+\mu) \pi)+\int_{(1+\mu) \pi}^{(2 n-1+\mu) \pi} t^{\mu-1} \sin (t-\mu \pi) d t \\
& \leq F_{\mu}((1+\mu) \pi),
\end{aligned}
$$

it suffices to prove that $F_{\mu}((1+\mu) \pi) \leq 0$ or, equivalently, that $z_{1} \leq(1+\mu) \pi$. However, since $\mu^{*}(\rho)=z_{1}^{(-1)}((\rho+1) \pi)$, the latter is equivalent to (3.3).

Proof of Theorem 1.2, It remains to verify (3.2) for $n \in\{2,3, \ldots\}$. Because of (3.1) we have

$$
\int_{0}^{\infty} t^{\mu-1} \sin (t-x) d t=\Gamma(\mu) \sin \left(\frac{\mu \pi}{2}-x\right), \quad x \in(0, \pi),
$$

and thus

$$
\int_{0}^{\infty} t^{\mu-1} \log (t) \sin (t-x) d t=\Gamma^{\prime}(\mu) \sin \left(\frac{\mu \pi}{2}-x\right)+\frac{\pi}{2} \Gamma(\mu) \cos \left(\frac{\mu \pi}{2}-x\right) .
$$


Since by Theorem $1.3 \mu \pi / 2<w_{2 n-1} \leq \mu \pi$, we find that

$$
J_{n}:=\int_{0}^{\infty} t^{\mu-1} \log (t) \sin \left(t-w_{2 n-1}\right) d t>0, \quad n \in \mathbb{N} .
$$

For $n \in \mathbb{N}$ define the sequence

$$
D_{n, k}:=\int_{\max \left\{0, w_{2 n-1}+(2 k-3) \pi\right\}}^{w_{2 n-1}+(2 k-1) \pi} t^{\mu-1} \log (t) \sin \left(t-w_{2 n-1}\right) d t, \quad k \in \mathbb{N} .
$$

Then

$$
\sum_{k=1}^{\infty} D_{n, k}=J_{n}>0
$$

and

$$
\sum_{k=1}^{n} D_{n, k}=\int_{0}^{z_{2 n-1}} t^{\mu-1} \log (t) \sin \left(t-w_{2 n-1}\right) d t
$$

The proof of the theorem will therefore be complete if we can show that for each $n \in\{2,3, \ldots\}$ there is a $k(n) \in \mathbb{N}$ such that $D_{n, k} \geq 0$ for $k \in\{1, \ldots, k(n)\}$ and $D_{n, k} \leq 0$ for $k>k(n)$.

It is easy to check that for every $\mu \in(0,1)$ there is a $t_{\mu}>e$ such that $t^{\mu-1} \log (t)$ is positive and increasing in $\left(1, t_{\mu}\right)$ and positive and decreasing in $\left(t_{\mu}, \infty\right)$. Let $k^{*} \in\{1,2,3, \ldots\}$ be such that $t_{\mu} \in\left[w_{2 n-1}+\left(2 k^{*}-3\right) \pi, w_{2 n-1}+\left(2 k^{*}-1\right) \pi\right)$. Then it is clear that $D_{n, k}>0$ for $k \in\left\{2, \ldots, k^{*}-1\right\}$ and $D_{n, k}<0$ for $k>k^{*}$. We therefore set $k(n)=k^{*}$ if $D_{n, k^{*}} \geq 0$ and $k(n)=k^{*}-1$ otherwise.

$D_{n, 1}>0$ remains to be verified. But since $\mu \pi / 2<w_{2 n-1} \leq \mu \pi$ for $\mu \in(0,1)$, this follows readily from Theorem 4.1 below.

We have thus shown that for $n \in \mathbb{N}$ the functions $z_{2 n-1}$ are strictly increasing in $(0,1)$. While the functions $z_{2 n}$ are not monotonic in $(0,1)$ for any $n \in \mathbb{N}$ (cf. Proposition 1.4), numerical computation strongly supports the conjecture that the functions $z_{2 n-1}$ and $z_{2 n}, n \in \mathbb{N}$, are convex and concave, respectively, in $(0,1)$. This, however, seems quite hard to prove.

\section{ON THE SIGN OF $I(\rho, \mu)$}

Recall that

$$
I(\rho, \mu):=\frac{d}{d \mu} F_{\mu}((\rho+1) \pi)=\int_{0}^{(\rho+1) \pi} t^{\mu-1} \sin (t-\rho \pi) \log (t) d t .
$$

In this section we will determine a large subset of $(\rho, \mu) \in(0,1)^{2}$ for which $I(\rho, \mu)$ is positive.

To that end, observe first that the function

$$
p(\mu):=\int_{0}^{\pi} t^{\mu-1} \log (t) \sin (t) d t
$$

is strictly increasing on $(0,1)$ and satisfies $p(0)=-0.53 \ldots$ and $p(1)=0.64 \ldots$. Consequently, the equation $p(\mu)=0$ has a unique solution $\mu_{0}$ in $(0,1)$. The numerical value of $\mu_{0}$ is $\mu_{0}=0.32 \ldots$. Further, let $M$ be the union of the three sets

$$
\begin{aligned}
& M_{1}:=\left\{(\rho, \mu):(10 \pi)^{-1} \leq \rho \leq 1,0 \leq \mu \leq 1\right\}, \\
& M_{2}:=\left\{(\rho, \mu): 0<\rho<(10 \pi)^{-1}, \mu_{0} \leq \mu \leq 1\right\}, \\
& M_{3}:=\left\{(\rho, \mu): 0<\mu \leq 2 \rho \leq(5 \pi)^{-1}\right\} .
\end{aligned}
$$


We will show the following.

Theorem 4.1. For all $(\rho, \mu) \in M$ we have $I(\rho, \mu)>0$. To each $\mu \in\left(0, \mu_{0}\right)$ there is a $\delta_{\mu}>0$ such that $I(\rho, \mu)<0$ for $\rho \in\left(0, \delta_{\mu}\right)$.

The second statement of this theorem has essentially been shown in the introduction. Hence, it only remains to verify that $I(\rho, \mu)>0$ for $(\rho, \mu) \in M$. To that end, we need some auxiliary lemmas. For $x \in[0, \pi]$ define

$$
f(x):=\int_{0}^{x} \log (t) \sin (t-x) d t \quad \text { and } \quad g(x):=\int_{0}^{\pi} \frac{\log (t+x)}{t+x} \sin (t) d t .
$$

Lemma 4.2. For $x \in(0,1)$ we have $f(x)>0$.

Proof. If $x \in(0,1)$, then clearly

$$
f(x) \geq \log (x) \int_{0}^{x} \sin (t-x) d t=-\log (x)(1-\cos (x))>0 .
$$

Lemma 4.3. The function $f(x)$ is concave on $(1, \pi)$.

Proof. We recall that

$$
\begin{aligned}
& \operatorname{Si}(x)=\int_{0}^{x} \frac{\sin (t)}{t} d t=\frac{\pi}{2}-\int_{x}^{\infty} \frac{\sin (t)}{t} d t \\
& \operatorname{Ci}(x)=-\int_{x}^{\infty} \frac{\cos (t)}{t} d t=\gamma+\log (x)-\int_{0}^{x} \frac{1-\cos (t)}{t} d t
\end{aligned}
$$

where $\gamma$ is Euler's constant.

It is then easy to see that

$$
\int_{0}^{x} \log (t) \cos (t) d t=\log (x) \sin (x)-\operatorname{Si}(x)
$$

and

$$
\int_{0}^{x} \log (t) \sin (t) d t=-\log (x) \cos (x)+\operatorname{Ci}(x)-\gamma .
$$

From these, the relation

$$
f(x)=-\log (x)+\cos (x)(\operatorname{Ci}(x)-\gamma)+\sin (x) \operatorname{Si}(x)
$$

follows. We observe also that

$$
f^{\prime \prime}(x)=-f(x)-\log (x) .
$$

Therefore

$$
f^{\prime \prime}(x)=\cos (x)(-\operatorname{Ci}(x)+\gamma)-\sin (x) \operatorname{Si}(x) .
$$

It is clear that the function $w(x):=-\mathrm{Ci}(x)+\gamma$ is strictly decreasing on $(0, \pi / 2)$ and strictly increasing on $(\pi / 2, \pi)$; hence $w(x) \geq w(\pi / 2)=0.1 \ldots>0$. Since $\operatorname{Si}(x)>0$ for all $x>0$, it readily follows from (4.3) that $f^{\prime \prime}(x)<0$ for $\pi / 2 \leq x \leq \pi$. On the other hand, for $1 \leq x<\pi / 2$ we have

$$
f^{\prime \prime}(x) \leq(-\operatorname{Ci}(1)+\gamma) \cos (x)-\operatorname{Si}(1) \sin (x)<0 .
$$

Lemma 4.4. The function $g(x)$ is concave on $(0, \pi)$. 
Proof. For $x \in(0, \pi)$ we have

$$
\begin{aligned}
g^{(4)}(x) & =\int_{0}^{\pi} \frac{24 \log (t+x)-50}{(t+x)^{5}} \sin (t) d t \\
& \leq(24 \log (2 \pi)-50) \int_{0}^{\pi} \frac{\sin (t)}{(t+x)^{5}} d t<0 .
\end{aligned}
$$

This implies $g^{\prime \prime \prime}(x)>g^{\prime \prime \prime}(\pi)=0.011 \ldots>0$, which, in turn, gives $g^{\prime \prime}(x)<g^{\prime \prime}(\pi)=$ $-0.0012 \ldots<0$ for $x \in(0, \pi)$.

We will now prove Theorem 4.1 separately for the three sets $M_{1}, M_{2}$, and $M_{3}$. Note that

$$
I(\rho, \mu)=\int_{0}^{\rho \pi} t^{\mu-1} \log (t) \sin (t-\rho \pi) d t+\int_{\rho \pi}^{(\rho+1) \pi} t^{\mu-1} \log (t) \sin (t-\rho \pi) d t .
$$

Proof of Theorem 4.1 for $(\rho, \mu) \in M_{1}$. It follows readily from (4.4) that for $(\rho, \mu) \in$ $M_{1}$,

$$
I(\rho, \mu) \geq f(\rho \pi)+g(\rho \pi) .
$$

It will therefore be enough to prove

$$
f(x)+g(x)>0 \quad \text { for } \quad x \in[1 / 10, \pi] .
$$

For $x \in\left[\frac{1}{10}, 1\right]$ we use Lemmas 4.2 and 4.4 to obtain

$$
f(x)+g(x)>g(x)>\min \{g(1 / 10), g(1)\}=g(1 / 10)=0.0819 \ldots>0 .
$$

For $x \in(1, \pi]$ Lemmas 4.3 and 4.4 give

$$
f(x)+g(x)>\min \{f(1)+g(1), f(\pi)+g(\pi)\}=f(\pi)+g(\pi)=0.0169 \ldots>0 .
$$

Proof of Theorem 4.1 for $(\rho, \mu) \in M_{2}$. Observe that for $\rho \in\left(0, \frac{1}{10 \pi}\right)$,

$$
\int_{0}^{\rho \pi} t^{\mu-1} \log (t) \sin (t-\rho \pi) d t>0 .
$$

Equation (4.4) therefore implies

$$
\begin{aligned}
I(\rho, \mu) & >\int_{\rho \pi}^{(\rho+1) \pi} t^{\mu-1} \log (t) \sin (t-\rho \pi) d t=\int_{0}^{\pi}(t+\rho \pi)^{\mu-1} \log (t+\rho \pi) \sin (t) d t \\
& \geq \int_{0}^{\pi}(t+\rho \pi)^{\mu_{0}-1} \log (t+\rho \pi) \sin (t) d t \geq \int_{0}^{\pi} t^{\mu_{0}-1} \log (t) \sin (t) d t=0
\end{aligned}
$$

for $\rho \in\left[0, \frac{1}{10 \pi}\right)$. Here we have made use of the fact that, for all $t \in(0, \pi)$, $(t+\rho \pi)^{\mu-1} \log (t+\rho \pi)$ is an increasing function of $\mu$ when $\rho \in\left[0, \frac{1}{10 \pi}\right)$ and an increasing function of $\rho$ when $\mu=\mu_{0}$.

Proof of Theorem 4.1 for $(\rho, \mu) \in M_{3}$. Since $\rho \in\left[\frac{\mu}{2}, \frac{1}{10 \pi}\right]$, we have

$$
\int_{0}^{(\rho+1) \pi} t^{\mu-1} \log (t) \sin (t-\rho \pi) d t>\int_{0}^{1} t^{\mu-1} \log (t) \sin (t-\rho \pi) d t .
$$


The integral on the right-hand side of this inequality can be written as

$$
\cos (\rho \pi) \int_{0}^{1} t^{\mu-1} \log (t) \sin (t) d t-\sin (\rho \pi) \int_{0}^{1} t^{\mu-1} \log (t) \cos (t) d t
$$

and is thus larger than

$$
\cos \left(\frac{\mu \pi}{2}\right) \int_{0}^{1} t^{\mu-1} \log (t) \sin (t) d t-\sin \left(\frac{\mu \pi}{2}\right) \int_{0}^{1} t^{\mu-1} \log (t) \cos (t) d t .
$$

Applying the estimates $\sin (t)<t$ and $\cos (t)>1-2 t / \pi, t \in(0, \pi / 2)$, and calculating the resulting integrals, we find that the term in (4.6) is larger than

$$
\frac{2 \mu \pi \sin \frac{\mu \pi}{2}-\pi \mu^{2} \cos \frac{\mu \pi}{2}+\sin \frac{\mu \pi}{2}\left(\mu^{2}(\pi-2)+\pi\right)}{\pi \mu^{2}(\mu+1)^{2}} .
$$

It is easy to check that $2 \sin \frac{\mu \pi}{2}>\mu \cos \frac{\mu \pi}{2}$ for $\mu \in\left(0, \frac{1}{5 \pi}\right]$, and therefore the proof is complete.

\section{REFERENCES}

1. J. Arias de Reyna and J. van de Lune, High precision computation of a constant in the theory of trigonometric series, Math. Comp. 78 (2009), no. 268, 2187-2191. MR.2521284 (2010j:65063)

2. G. Brown, K. Y. Wang, and D. C. Wilson, Positivity of some basic cosine sums, Math. Proc. Cambridge Philos. Soc. 114 (1993), no. 3, 383-391. MR.1235986 (94h:42013)

3. M. L. Glasser, Integral representations for the exceptional univariate Lommel functions, J. Phys. A 43 (2010), no. 15, 155207, 4 pp. MR2608275 (2011e:33011)

4. S. Koumandos, An extension of Vietoris's inequalities, Ramanujan J. 14 (2007), no. 1, 1-38. MR $2298638(2008 \mathrm{e}: 42003)$

5. S. Koumandos and M. Lamprecht, Complete monotonicity and related properties of some special functions, Math. Comp., to appear.

6. S. Koumandos and S. Ruscheweyh, Positive Gegenbauer polynomial sums and applications to starlike functions, Constr. Approx. 23 (2006), no. 2, 197-210. MR2186305 (2007b:42001)

7. 149 (2007), no. 1, 42-58. MR2371613 (2009f:42001)

8. E. Kreyszig, Über den allgemeinen Integralsinus Si $(z, \alpha)$, Acta Math. 85 (1951), 117-181. MR.0041287 (12:825a)

9. J. Steinig, The sign of Lommel's function, Trans. Amer. Math. Soc. 163 (1972), 123-129. MR0284625(44:1849)

10. G. N. Watson, A Treatise on the Theory of Bessel Functions, Cambridge University Press, Cambridge, England, 1944. MR0010746 (6:64a)

11. A. Zygmund, Trigonometric series. Vols. I, II, third ed., Cambridge Mathematical Library, Cambridge University Press, Cambridge, 2002. MR.1963498(2004h:01041)

Department of Mathematics and Statistics, The University of Cyprus, P. O. Box 20537, 1678 Nicosia, Cyprus

E-mail address: skoumand@ucy.ac.cy

Department of Mathematics and Statistics, The University of Cyprus, P. O. Box 20537, 1678 Nicosia, Cyprus

E-mail address: martin@ucy.ac.cy 